\title{
Diseño de un plan de formación en competencias informacionales en los estudios de grado
}

\author{
Disigning an information literacy programme for undergraduate studies
}

\begin{abstract}
Matilde CATín Luna (1), Javier Vidal Bordes (2), Elena Escar HERnÁndez (3), Genaro LAMARCA LANGA (4), Luisa Orera Orera (5), José Antonio Salvador Oliván (6)

(1) Biblioteca de Humanidades María Moliner; (2) Biblioteca Universitaria de Zaragoza; (3) Biblioteca de la Escuela Universitaria de Zaragoza (4) (5) (6) Departamento de Ciencias de la Documentación e Historia de la Ciencia, Universidad de Zaragoza, Pedro Cerbuna 12; 50009 Zaragoza; lamarca@unizar.es.
\end{abstract}

\begin{abstract}
Resumen
Avance de la Memoria del Proyecto denominado "Diseño de un plan de formación en competencias informacionales en los estudios de grado", que forma parte de las Convocatorias de Innovación Docente 2009/2010 de la Universidad de Zaragoza, PIECyT 2009. Se explica la metodología del mismo así como el diseño del plan y sus tres niveles de desarrollo.
\end{abstract}

Palabras clave: Competencias informacionales. Alfabetización informacional. Formación de usuarios. Estudios de grado. Bibliotecas universitarias. Universidad de Zaragoza.

\section{Introducción}

La normativa legal que organiza el Espacio Europeo de Educación Superior en España tiende a favorecer en los nuevos grados la incorporación de diferentes competencias transversales, entre ellas, y en lugar muy destacado, promueve la habilidad en la búsqueda, el análisis y la gestión de la información, es decir las competencias informacionales $(\mathrm{Cl})$, como se recoge en las recomendaciones de los libros blancos de los títulos de grado de la ANECA.

En algunos países esas $\mathrm{Cl}$ están plenamente integradas dentro de los diferentes estudios. En España no lo ha sido tanto, y en concreto en la Universidad de Zaragoza esa integración, dentro de los diferentes planes de estudios, ha sido muy escasa.

Esta situación necesita ser conocida y corregida en la medida que sea posible. Por ello la Biblioteca de la Universidad de Zaragoza, (a través de la Biblioteca María Moliner de la Facultad de Filosofía y Letras, y de la Biblioteca de la Escuela Politécnica Superior en Huesca) y profesores del Grado en Información y Documentación, miembros del Departamento de Ciencias de la Documentación e Historia de la Ciencia, presentamos en el curso 2009-2010 un proyecto de-

\begin{abstract}
Preliminary version of Project Report titled "Design of an education plan on informational literacy competencies in degree studies," which is part of the Teaching Innovation Call for 2009/2010 at the University of Zaragoza, PIECYT 2009. It also explains the methodology and the design of the plan and its three levels of development.
\end{abstract}

Keywords: Information literacy. Information competences. Training. Higher education. Undergraduate studies. University Libraries. Zaragoza (Spain).

nominado "Diseño de un plan de formación en competencias informacionales en los estudios de grado" para colaborar en la corrección de este problema.

\section{Objetivo}

El Objetivo del trabajo, es coincidente con el objetivo del Proyecto y es la elaboración de un plan de formación en $\mathrm{Cl}$, dividido en tres niveles a desarrollar a lo largo de los distintos grados y adaptado a las diferentes áreas de estudio, que sirva de guía general para que, desde las distintas bibliotecas de la Universidad de Zaragoza se pueda llevar a cabo un proceso integral de enseñanza de $\mathrm{Cl}$, adaptadas a los estudiantes de los grados impartidos en nuestra universidad.

Dicho plan se plasmará en la elaboración de una guía dotada de una gran flexibilidad de contenidos que permita ser utilizada por todos los grados, teniendo en cuenta la heterogeneidad de estos, así como la de los centros donde se imparten.

El diseño de esta guía supone la elaboración de un programa ALFIN, que ayudará a que la Biblioteca Universitaria de Zaragoza se convierta en un centro de recursos para el aprendizaje y la investigación (CRAI). 


\section{Metodología}

El método de trabajo se ha desarrollado en reuniones quincenales de grupo y en tareas individuales. Todas las decisiones han sido tomadas por consenso entre los miembros del grupo.

El método seguido para la elaboración del plan de formación de $\mathrm{Cl}$ en los estudios de grado de la UZ ha constado de las siguientes fases:

1. Planificación

2. Recogida de documentación

3. Análisis de titulaciones

4. Normalización

5. Diseño del plan

\subsection{Planificación}

Desde el primer momento nuestra experiencia docente y de formación de usuarios nos hizo tener claro que debíamos ser prácticos y realistas a la hora de elaborar un plan de formación en $\mathrm{Cl}$. Era imprescindible conocer de dónde partíamos así como saber a dónde queríamos llegar, por supuesto contando con el apoyo de las autoridades académicas y de la dirección de la Biblioteca Universitaria.

El plan debía ser global (alcanzando a todos los grados y desde todas las bibliotecas), debería contar con el apoyo institucional (Vicerrectorado y Coordinadores de Grado) y de integración de las actividades en los planes de estudio.

Tras un debate apostamos por seguir la metodología que pasamos a desarrollar en los siguientes puntos.

\subsection{Recogida de documentación}

Nos planteamos en primer lugar la necesidad de señalar qué $\mathrm{y}$, cuáles son las $\mathrm{Cl}$ requeridas en un nivel de enseñanza superior, para posteriormente valorar de qué modo aparecen recogidas en las asignaturas que componen los distintos grados que se imparten o van a impartirse a partir del curso 2010-2011 en la Universidad de Zaragoza.

Para la selección de las $\mathrm{Cl}$, revisamos los siguientes documentos: la Guía de buenas prácticas para el desarrollo de las competencias informacionales en las Universidades Españolas realizada por el Grupo de Trabajo ALFIN, el documento Competencias informáticas e informacionales en los estudios de Grado, la Jornada de Trabajo de responsables de ALFIN en Bibliotecas Universitarias Españolas y la relativa a formación de usuarios en bibliotecas de las Universidades de Sevilla y Zaragoza.

El punto de partida para el establecimiento de las $\mathrm{Cl}$ lo constituye el documento antes mencionado, elaborado por la Comisión Mixta CRUETIC REBIUN, en abril de 2009, titulado Competencias informáticas e informacionales en los estudios de Grado, en el que se señalan los siguientes descriptores para el bloque temático informacional:

1. Proceso de búsqueda

2. El catálogo de la biblioteca

3. Los recursos electrónicos

4. La evaluación de la información

5. Cómo citar recursos de información

6. Cómo redactar un trabajo

7. Los derechos de autor

8. Peligros y riesgos de la red

A partir de estos descriptores pasamos a la siguiente fase de análisis de la situación de las $\mathrm{Cl}$ en las Memorias de Grado de la Universidad de Zaragoza.

\subsection{Análisis de titulaciones}

Para llevar a cabo el análisis del tratamiento dado a las $\mathrm{Cl}$ en los 54 grados implantados o a implantarse en el curso 2010-2011 en la Universidad de Zaragoza, se reúnen las Memorias de grado, que revisamos detenidamente, excluyendo del análisis el Grado Información y Documentación, ya que dichas $\mathrm{Cl}$ forman parte de los contenidos de sus asignaturas.

Para facilitar la tarea de revisión de las titulaciones y lograr una mayor homogeneidad en la recogida y posterior tratamiento de los datos, elaboramos una base de datos en FileMaker, con la siguiente estructura y campos:

1. Nombre del Grado: Campo que recoge la denominación del Grado.

2. Tipo de competencia: Se distingue entre General, específica y específica de materia/asignatura.

3. Denominación de la competencia: Se recoge la competencia tal y como está expresada en la Memoria.

4. Materia/Asignatura: Contiene el nombre de la materia o asignatura en la que aparece la competencia o competencias.

5. Tipo de asignatura: Se distingue entre obligatoria, optativa y formación básica. 
6. Créditos: Señala los créditos de las diferentes asignaturas.

El análisis de las memorias supone un arduo trabajo individual y una constante puesta en común de los datos entre los miembros del grupo para decidir, en caso de duda, si determinadas competencias, tal como se expresan en las asignaturas, son o no informacionales. Ante el variado número y modo en que se expresan estas competencias se procede a su revisión, normalización e inclusión en la base de datos.

La disparidad observada en la expresión de las $\mathrm{Cl}$ en las Memorias de los Grados, nos obliga a unificarlas y agruparlas de forma sintetizada en una nueva base de datos, que nos va a permitir establecer una relación entre la forma como aparecen en las Memorias y las propuestas realizadas por el grupo de trabajo.

Tras un largo debate en sucesivas reuniones acordamos adoptar las denominaciones siguiendo el documento elaborado por la Comisión mixta CRUE-TIC y REBIUN, de abril de 2009 (1).

\subsection{Normalización}

Una vez establecida la relación antes indicada y dado el diferente modo con el que, en las Memorias, se hace referencia a una misma $\mathrm{Cl}$, vimos necesario proponer su normalización.

Como resultado de este análisis llegamos a la conclusión de que había que establecer una $\mathrm{Cl}$ que resumiera y sintetizase todos los contenidos expuestos para que, en adelante, pudiera ser utilizada de forma unívoca, sin dar lugar a equívocos, en el momento de la revisión de las memorias de verificación de los grados, en la elaboración de las guías académicas, o documentos similares.

En este sentido, proponemos a la Universidad de Zaragoza como competencia normalizada y unificada, para su uso en todos los grados, la competencia general que se denominará: Buscar, gestionar y utilizar la información, que se desarrolla en las siguientes competencias específicas:

\section{Conocer fuentes de información}

2. Seleccionar las fuentes pertinentes.

3. Analizar, evaluar y elegir las fuentes de información pertinentes.

4. Buscar y recuperar información.

5. Evaluar la información.

6. Tratar y organizar la información.
7. Presentar la información de forma estandarizada.

8. Comunicar y difundir la información.

\subsection{Diseño del plan}

Para desarrollar el contenido de la competencia general y las específicas antes señaladas, se toma como modelo el utilizado en la Universidad de Zaragoza para la elaboración de las guías docentes de las diferentes asignaturas de grado, ya que obedece a los modernos planteamientos del Espacio Europeo de Educación Superior.

En el diseño del plan de formación y en aras de un mayor aprovechamiento de los recursos, se tienen en cuenta, entre otras, las experiencias en formación de usuarios que se han llevado a cabo en la Universidad de Zaragoza, como es el caso del Plan de Formación de usuarios de la BUZ o el Proyecto de Formación realizado para el Grado en Ciencias Ambientales de la Escuela Politécnica de Huesca, denominado Actividades encaminadas a la adquisición de la competencia transversal "Gestión de la información".

\section{Resultados: el Plan de formación en competencias informacionales}

Una vez analizada la situación de la UZ, siguiendo las Memorias del nuevo modelo educativo y en concreto en la formación en las competencias transversales informacionales, articulamos un procedimiento coordinado para que el estudiante adquiera una formación en competencias que sea gradual, ligado a una asignatura troncal, por lo que se establecen tres niveles de aprendizaje progresivo para cada una de las competencias específicas indicadas anteriormente:

1. Básico: orientado a los estudiantes de primer curso

2. Medio: orientado a los estudiantes de segundo o tercer curso

3. Avanzado: orientado a los estudiantes de último curso

Siguiendo el modelo de elaboración de las guías docentes establecemos los contenidos de cada competencia en relación con estos tres niveles, comenzando por el nivel básico, y atendiendo a los resultados de aprendizaje, en este caso nos planteamos: ¿qué debe saber el estudiante de primer curso de la UZ para ser competente en la búsqueda, gestión y utilización de la información? Esta pregunta será la misma en los niveles medio y avanzado. 


\subsection{Nivel básico}

En el nivel básico se proponen los siguientes contenidos en relación con las competencias propuestas:

1. Conocer y utilizar fuentes de información:

- La web de la biblioteca como plataforma de acceso a los recursos de información.

- Bibliografía recomendada y el catálogo (webopac).

- Dialnet y bases datos del CSIC.

2. Buscar y recuperar información:

- Tipología documental (conceptos básicos, contenidos en cada tipo de fuente, dando mayor importancia a la tipología que al soporte).

- Buscar en el catálogo (webopac).

3. Valorar y evaluar la información:

- Diferenciar entre la información evaluada de la no evaluada.

- Valorar la biblioteca como recurso web de valor añadido: selección de recursos, recursos de pago, etc.

4. Gestionar la información:

- Cómo interpretar una referencia bibliográfica (una monografía, parte de una monografía, un artículo de revista, un recurso electrónico, etc.), enfocado de forma sencilla, utilizando ejemplos.

- Conocer la existencia de gestores bibliográficos.

- Cómo hacer una bibliografía, con ejemplos básicos (monografía, parte de una monografía, artículo de revista, página web).

5. Comunicar y difundir la información:

- Cómo se hace un trabajo académico

\subsection{Nivel medio}

Los contenidos del nivel medio se centran en los siguientes puntos:

1. Conocer y utilizar fuentes de información: fuentes generales de información y motores de búsqueda.

2. Buscar y recuperar información: diseño de estrategias de búsqueda.

3. Evaluación de la información: criterios de contenido.
4. Gestionar la información: Utilización de Refworks, organización y presentación de bibliografías.

\subsection{Nivel avanzado}

Los contenidos y competencias del tercer nivel se desarrollan en la asignatura "Trabajo fin de grado" y son:

1. Conocer y utilizar fuentes de información: las fuentes específicas han de tener relación con las titulaciones, en función de las macroáreas, áreas o grados. En este sentido deberán seleccionarse las bases de datos de la BUZ así como otras de carácter gratuito. Se señalan como fundamentales las bases de datos del ISI.

2. Buscar y recuperar información: técnicas de búsqueda específicas.

3. Valorar y evaluar: conocer qué es y para qué se utiliza el factor de impacto y el índice $\mathrm{H}$.

4. Gestionar la información: Utilización avanzada de Refworks.

5. Comunicar y difundir la información, adaptada a los diferentes grados.

\section{Acreditación}

Un aspecto que no podemos olvidar es el que se refiere a la certificación; se han barajado varios tipos a aplicar en cada uno de los niveles de aprendizaje:

1. La acreditación de la formación mediante un certificado del Vicerrectorado para que conste en el expediente del estudiante que ha realizado un curso básico ligado a una determinada asignatura.

2. Un crédito para el nivel medio ligado a una asignatura (entendida la formación como una actividad académica complementaria).

3. Para el nivel superior, el reconocimiento de la acreditación en la evaluación de la asignatura del "Trabajo fin de grado".

Todos estos reconocimientos aparecerán reflejados en el expediente académico del estudiante.

\section{Conclusiones}

Entendemos que este es el momento idóneo para la implantación del plan propuesto ya que todos los centros de la Universidad de Zaragoza cuentan con un primer curso de grado y personal bibliotecario formado en $\mathrm{Cl}$. Señalar que en el mapa de procesos de la BUZ la formación de usuarios en un proceso clave y que está previs- 
to para el comienzo de curso 2010-2011 ofrecer un curso semipresencial para todos los estudiantes de primer curso de grado a través de la plataforma docente Moodle. De esta manera se hace posible uno de los objetivo del Plan Estratégico 2009-2012 de la BUZ que procura la "integración de la oferta formativa de la biblioteca en los planes educativos de la Universidad de Zaragoza".

En cuanto a la viabilidad del Plan queremos destacar que desde el primer momento se trabaja de forma coordinada con el grupo de trabajo de formación de usuarios de la BUZ, y en comunicación con el Adjunto al Rector para Innovación Docente.

Estamos convencidos de que la formación en $\mathrm{Cl}$ es una necesidad para el estudiante universitario y que la Universidad debe atender para que sea una realidad, por otra parte también que la Biblioteca Universitaria debe colaborar con los docentes en la formación en estas competencias transversales en la línea de convertirse en un auténtico centro de recursos para el aprendizaje y la investigación.

\section{Notas}

(1) Se describen las siguientes competencias informacionales:
(a) Conocer y utilizar fuentes de información
(b) Buscar y recuperar información
(c) Analizar y seleccionar la información
(d) Valorar y evaluar la información
(e) Gestionar la información
(f) Comunicar y difundir la información

\section{Referencias}

Biblioteca de la Universidad de Sevilla. Programa de Formación en Competencias Informacionales de la Universidad de Sevilla. http://bib.us.es/aprendizaje investigacion /formacion/common/programa_fu.pdf (2010-02-14).

Biblioteca de la Universidad de Zaragoza. Plan de formación de usuarios de la BUZ. Resultados e indicadores. Curso 2008/2009, Curso 2009/2010 (Primer cuatrimestre). Intranet de la BUZ. (2010-02-24).

Biblioteca de la Universidad de Zaragoza. Plan de formación en competencias informacionales de la BUZ: Informe para la Comisión técnica. Intranet de la BUZ. (2010-02-24).

Comisión mixta CRUE-TIC y REBIUN. (2009). Competencias informáticas e informacionales en los estudios de Grado.

$<$ http://www.rebiun.org/doc/documento competencias in formaticas.pdf> (2010-02-18).

Grupo de trabajo ALFIN REBIUN (2009). Jornada de Trabajo de Responsables de ALFIN en las bibliotecas universitarias españolas. Ministerio de Cultura. http:// www.rebiun.org/export/docReb/jornada_trabajo_alfin.doc (2010-02-20).
Paricio Royo, J. (2010). Un modelo de guía docente desde los resultados de aprendizaje y su evaluación. Zaragoza: Universidad de Zaragoza, Instituto de Ciencias de la Educación, 2010.

REBIUN. Grupo de Trabajo ALFIN (2008). Guía de buenas prácticas para el desarrollo de las competencias informacionales en las Universidades Españolas. http:// www.rebiun.org/export/docReb/guia_buenas_practicas. doc (2010-02-10).

Recibido: 15-06-2010.

Aceptado: 15-06-2010. 
